\title{
Etude électrophorétique de la protéolyse au cours de l'affinage des fromages à pâte persillée du type bleu d'Auvergne
}

\author{
par \\ P. TRIEU-CUOT et J.C. GRIPON
}

\section{Rés u m é}

L'évolution de la protéolyse au cours de l'affinage de bleus d'Auvergne a été suivie par électrophorèse, focalisation isoélectrique et électrophorèse bidimensionnelle.

L'importance des dégradations produites par le Penicillium roqueforti est clairement mise en évidence. Les actions respectives de la métalloprotéase et de l'aspartyle protéase excrétées par la moisissure ont été caractérisées et suivies à l'aide de produits de dégradation de la caséine $\beta$ utilisés comme marqueurs. Ces deux enzymes sont actives dès le développement du Penicillium. Ultérieurement, l'intensité de coloration des produits résultant de la métalloprotéase diminue alors que celle d'un produit majeur résultant de l'aspartyle protéase augmente, suggérant que cette enzyme poursuit son action jusqu'à 30 jours d'affinage au moins.

Les diagrammes électrophorétiques étudiés révèlent que, qualitativement, la protéolyse développée par le $P$. roqueforti dans les pâtes persillées est très voisine de celle due au $P$. caseicolum dans les fromages à croûte fleurie (J. Dairy Res. (1982), 49, 501-510). La protéase alcaline du lait semble moins active dans les bleus d'Auvergne étudiés que dans les fromages de type camembert probablement parce que le $\mathrm{pH}$ de la pâte est moins élevé.

Mots clés :

Affinage - Protéolyse - Pâte persillée - Bleu d'Auvergne - Penicillium roqueforti Métalloprotéase - Aspartyle protéase - Electrophorèse - Focalisation isoélectrique - Electrophorèse bidimensionnelle.

Titre abrégé :

Protéolyse au cours de l'affinage des pâtes persillées.

Laboratoire de Biochimie et Technologie Laitières, Institut National de la Recherche Agronomique, C.N.R.Z. - 78350 Jouy-en-Josas (France). 


\section{S u m m a ry}

ELECTROPHORETIC STUDY OF PROTEOLYSIS DURING RIPENING OF BLUE CHEESE (BLEU D'AUVERGNE TYPE)

Electrophoresis, isoelectric focusing and 2-dimensional electrophoresis were used to study the development of proteolysis during «bleu d'Auvergne» ripening.

The importance of modifications due to Penicillium roqueforti was clearly evidenced. The respective activities of the metallo- and aspartyl-proteinases synthesized by this mold were characterized and followed using $\beta$-casein degradation products as marquers. The activities of the two enzymes were detectable immediatly after the appearance of the Penicillium. There after the amount of $\beta$-casein degradation peptides resulting from the metalloproteinase decreased while that of a major product resulting from the aspartyl proteinase increased suggesting that this enzyme is still active at least after 30 d. of ripening.

The studied electrophoretic diagramms revealed qualitatively that the proteolysis due to $\mathrm{P}$. roqueforti in blue cheese is very similar to that due to P. caseicolum in white molded cheese such as camembert (J. Dairy Res. (1982), 49, 501-510). The alkaline milk proteinase seemed to be less active in the "bleu d'Auvergne» than in camembert type cheese probably because the $\mathrm{pH}$ of the curd is lower.

Key words:

Ripening - Proteolysis - Blue cheese - Bleu d'Auvergne - Penicillium roqueforti - Metalloproteinase - Aspartyl proteinase - Electrophoresis - Isoelectric focusing - 2-dimensional electrophoresis.

Running title:

Proteolysis during blue cheese ripening.

\section{INTRODUCTION}

La protéolyse développée dans les fromages à pâte persillée est importante; dans un roquefort en fin d'affinage, l'azote soluble représente environ $50 \%$ de l'azote total (Devoyod et al., 1968) alors que par comparaison, il n'atteint que $30-35 \%$ en surface d'un fromage à croûte fleurie tel que le camembert (Lenoir, 1962). L'utilisation de caillés à flore contrôlée (Desmazeaud et al., 1975) a permis de montrer que, en dépit de la présence d'une microflore variée, la protéolyse est, pour la plus grande part, due à l'activité de la moisissure $P$. roqueforti. Le système protéolytique exocellulaire de $P$. roqueforti est analogue à celui de $P$. caseicolum, utilisé pour la fabrication des fromages à croûte fleurie. Ces deux moisissures 
synthétisent et excrètent des exopeptidases, une aspartyle protéase (EC 3.4.23.6) et une métalloprotéase (EC 3.4.24.4). L'action de ces 2 dernières enzymes sur les caséines a été étudiée (Trieu-Cuot et al., 1982 a et b) et l'on a constaté que les protéases homologues de ces 2 Penicillia ont des actions très voisines sur les caséines.

L'objet du présent mémoire est de suivre et de caractériser l'action des différentes protéases (chymosine, plasmine, aspartyle et métalloprotéase de $P$. roqueforti) susceptibles de provoquer de profondes modifications de la fraction azotée d'un fromage à pâte persillée tel que le Bleu d'Auvergne. Cette caractérisation a été effectuée par électrophorèse, focalisation isoélectrique et électrophorèse bidimensionnelle en utilisant essentiellement les produits de dégradation de la caséine $\beta$ comme marqueurs de l'activité des différentes enzymes ainsi que nous l'avions fait précédemment au cours d'une étude analogue sur le camembert (Trieu-Cuot et Gripon, 1982).

\section{MATERIEL ET METHODES}

\section{Fabrication des fromages}

Les Bleus d'Auvergne ont été fabriqués selon une technologie classique (Vesseyre, 1975). Les variants génétiques des caséines du lait de fabrication étaient les suivants : $\alpha_{s_{1}} \mathrm{~B}, \beta \mathrm{A}^{1}$ (principalement) et $\beta \mathrm{A}^{2}$, KA et $\mathrm{KB}$.

Le $P$. roqueforti utilisé était la souche PV (Centro Sperimentale del Latte, Milan). L'affinage était suivi pendant une période de 59 jours.

\section{Préparation des échantillons - mesure du $\mathrm{pH}$}

Les échantillons ont été prélevés $8,13,17,29$ et 59 jours après la fabrication. Le prélèvement soumis aux électrophorèses correspondait à la section aa', bb', $\mathrm{cc}^{\prime}$ ainsi qu'il est indiqué figure 1 . La préparation des solutions de fromage et des fractions insolubles à $\mathrm{pH}$ 4,6 a été décrite précédemment (Gripon et al., 1975). Le pH des fromages était mesuré à l'aide d'une micro électrode de pénétration de $3 \mathrm{~mm}$ de diamètre en 5 points d'un secteur du fromage comme il est décrit figure 1.

\section{Techniques électrophorétiques}

Les électrophorèses étaient effectuées dans un gel d'acrylamide-bisacrylamide-agarose $(5-0,5-0,8 \%)$ en tampon Tris-glycine $(0,075 \mathrm{M}-0,06 \mathrm{M} ; \mathrm{pH} 8,6)$ contenant $4,5 \mathrm{M}$ d'urée et $0,2 \%$ de $\beta$-mencaptoéthanol. Les focalisations isoélectriques étaient réalisées en gel d'acrylamide-bisacrylamide $(5-0,5 \%)$ contenant $2 \%$ d'ampholytes 


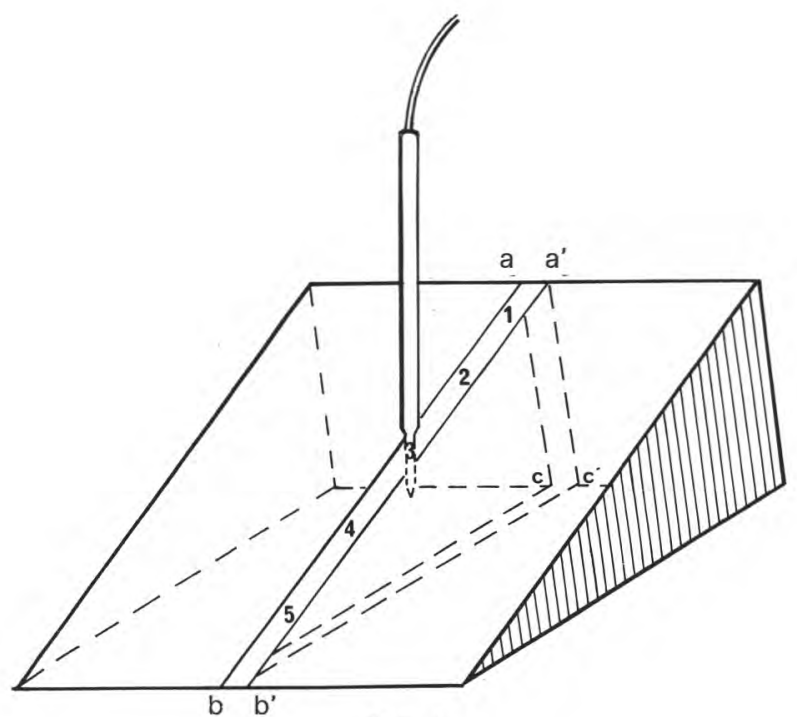

Fig. 1

Représentation schématique d'une tranche de bleu d'Auvergne. La protéolyse était étudiée sur la section ( $\left(a^{\prime}, b^{\prime}, c^{\prime}\right)$. Le $\mathrm{pH}$ était mesuré aux points 1, 2, 3, 4 et 5 avec une micro-électrode de pénétration.

Schematic drawing of a sector of bleu d'Auvergne. Proteolysis was studied on the volume $\left(a a^{\prime}, b b^{\prime}, c c^{\prime}\right)$. $p H$ was measured in position 1, 2, 3, 4 and 5 with a spear-type electrode.

(Bio-Lyte 4-9 technical grade) et de l'urée 7M. La première dimension de l'électrophorèse bidimentionnelle était une focalisation isoélectrique et la seconde une électrophorèse réalisée dans un gel à gradient d'acrylamide-bisacrylamide $(16-0,4 \% / 28-0,7 \%)$ en tampon Tris $\mathrm{HCl}(0,675 \mathrm{M}, \mathrm{pH} 8,8)$ contenant $4,9 \mathrm{M}$ d'urée et $0,1 \%$ de dodécylsulfate de sodium.

Ces techniques ont été conduites selon des conditions précédemment décrites (Gripon et al., 1975 ; Trieu-Cuot et Gripon, 1981 a).

\section{Identification des différents produits de dégradation}

L'étude de l'action de la chymosine, de la pepsine bovine A (Trieu-Cuot, 1981), de la plasmine (Trieu-Cuot et Gripon, 1981 a), de l'aspartyle protéase et de la métalloprotéase de $P$. roquefort $i$ (Trieu-Cuot et al., 1982 a et b) sur les caséines purifiées a permis l'identification des produits de dégradation présents sur les diagrammes électrophorétiques étudiés. 
TABLEAU 1 - TABLE 1

Evolution du $\mathrm{pH}$ au cours de l'affinage - Développement du Penicillium

Le développement du Penicillium était apprécié visuellement par l'apparition d'une coloration verte.

(0) absence de coloration, $(+)(++)(+++)$ coloration d'intensité croissante

$p H$ development during cheese ripening - Penicillium growth

The growth of the Penicillium was visually estimated by the appearance of a green color. (0) no colouration, $(+)(++)(+++)$ colouration with increasing intensity

\begin{tabular}{|c|c|c|c|c|c|c|}
\hline Emplacement de la mesure & $8 \mathrm{j}$ & $13 \mathrm{j}$ & $17 \mathrm{j}$ & $21 \mathrm{j}$ & $29 \mathrm{j}$ & $59 \mathrm{j}$ \\
\hline 1 & $5,05(0)$ & $5,03(0)$ & $5,13(0)$ & $5,33(0)$ & $5,44(0)$ & $6,24(0)$ \\
\hline 2 & $5,04(0)$ & $5,15(0)$ & $5,27(+)$ & $5,26(0)$ & $5,58(+)$ & $6,14(+++)$ \\
\hline 3 & $4,96(0)$ & $5,04(0)$ & $5,53(++)$ & $5,43(++)$ & $5,96(++)$ & $6,11(+++)$ \\
\hline 4 & $4,94(0)$ & $5,06(0)$ & $5,56(++)$ & $5,68(++)$ & $5,88(++)$ & $6,21(+++)$ \\
\hline 5 & $5,07(0)$ & $5,04(0)$ & $5,65(++)$ & $5,67(++)$ & $6,03(++)$ & $6,20(+++)$ \\
\hline
\end{tabular}




\section{RESULTATS}

\section{Evolution du $\mathrm{pH}$ au cours de l'affinage (tableau 1)}

Celui-ci augmente sensiblement entre 13 et 17 jours d'affinage, notamment au centre du fromage, au moment où le développement du $P$. roqueforti devient visible à l'œil nu. Les valeurs mesurées après 29 et 59 jours d'affinage (6,03 et 6,20 dans la partie centrale) sont inférieures à celles observées dans la zone externe d'un camembert affiné (Trieu-Cuot et Gripon, 1982).

\section{Etude par électrophorèse à $\mathrm{pH}$ alcalin}

Huit jours après la fabrication, alors que le Penicillium ne s'est pas encore développé, une fraction notable de la caséine $\alpha_{S_{1}}$ est dégradée en un peptide plus mobile $\alpha_{s_{1} I}$. Une modification importante des diagrammes électrophorétiques intervient après 17 jours d'affinage, c'est-à-dire juste après le développement du Penicillium. De nouvelles bandes de faible mobilité électrophorétique résultant de l'hydrolyse de la caséine $\beta$ ( $\beta \mathrm{mpla}+$ $\beta \mathrm{mp} 1 \mathrm{~b}),(\beta \mathrm{mp} 2 \mathrm{a}+\beta \mathrm{ap} 1)$ apparaissent; d'autres bandes s'intensifient $\left(\gamma_{3}+\beta \mathrm{mp} 3\right),\left(\gamma_{1}+\beta \mathrm{mp} 4\right),(\beta \mathrm{mp} 5+\beta \mathrm{ap} 3)$ (fig. 2). La bande

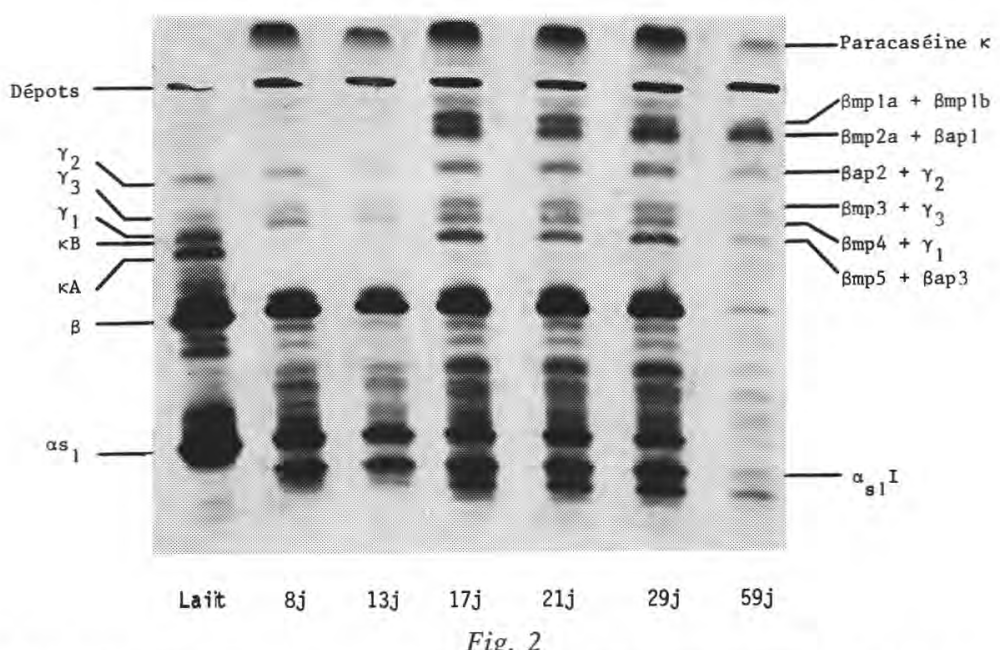

Evolution des diagrammes électrophorétiques au cours de l'affinage (fraction insoluble à pH 4,6).

Development of electrophoretic patterns during bleu d'Auvergne ripening (pH 4.6 insoluble protein).

Abréviations : $\beta \mathrm{mp}$ : produit d'hydrolyse de la caséine $\beta$ par la métalloprotéase : $\beta$ ap : produit d'hydrolyse de la caséine $\beta$ par l'aspartyle protéase ; $\gamma$ : produit d'hydrolyse de la caséine $\beta$ par la plasmine. 

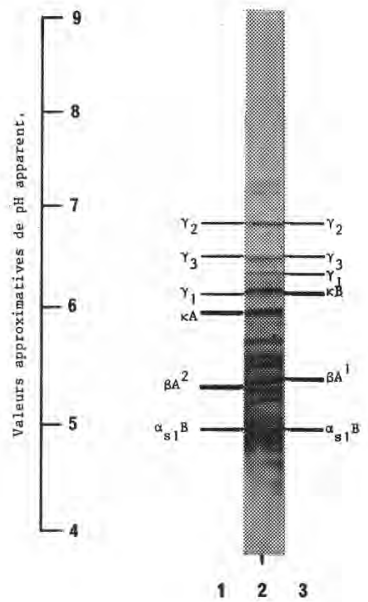
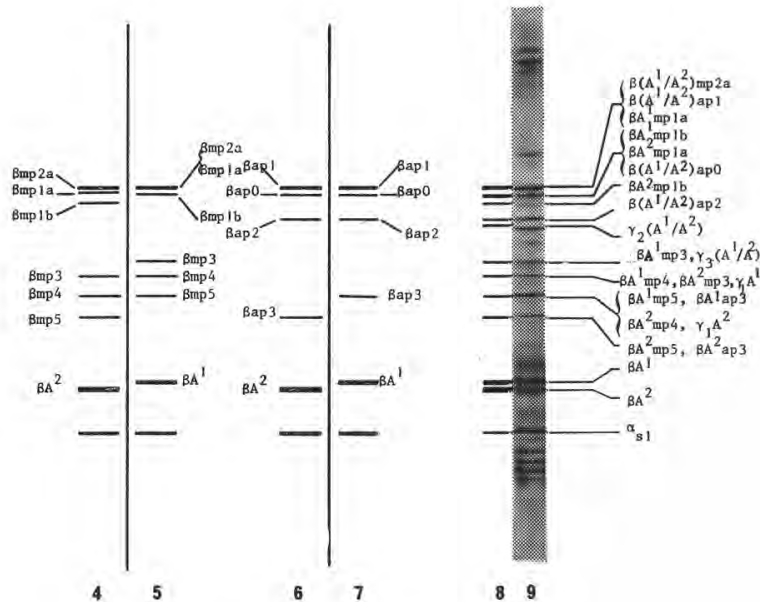

Fig. 3

Diagrammes de focalisation isoélectrique d'hydrolysats de caséines : 1 : Caséine bovine entière $\left(\alpha_{s_{1}} B, \beta A^{2}, K A\right) 2$ : lait de fabrication ; 3 : caséine bovine entière $\left(\alpha_{s_{1}} B, \beta A^{1}, K B\right) ; 4$ et 5 : respectivement caséine $\beta A^{2}$ et $\beta A^{1}$ hydrolysée par la métalloprotéase de Penicillium roqueforti ; 6 et 7 : respectivement caséine $\beta \mathrm{A}^{2}$ et $\beta \mathrm{A}^{1}$ hydrolysée par l'aspartyle protéase de $P$. roqueforti ; 8 : superposition des diagrammes $1,3,4,5,6,7 ; 9$ : fraction insoluble à $\mathrm{pH} 4,6$ d'un bleu d'Auvergne après 29 jours d'affinage.

Isoelectric focusing patterns of hydrolysates of caseins: 1: whole bovine casein $\left(\alpha_{s 1} B, \beta A^{2}, K A\right) ; 2$ : milk used for cheesemaking: 3: whole bovine casein ( $\alpha_{S 1}$, $\beta A^{1}$, $K B) ; 4$ and 5: respectively $\beta A^{2}$ and $\beta A^{1}$ casein hydrolysed by Penicillium roqueforti metalloproteinase; 6 and 7: respectively $\beta A^{2}$ and $\beta A^{1}$-casein hydrolysed by $P$. roqueforti aspartyl proteinase; 8: superposition of patterns $1,3,4,5,6,7 ; 9: \mathrm{pH}$ 4.6 insoluble fraction of bleu d'Auvergne at 29 days of ripening.

$(\beta m p 1 a+\beta m p 1 b)$ qui est un marqueur de l'activité de la métalloprotéase et la bande ( $\beta$ mp2a $+\beta a p 1)$ qui est principalement due à l'action de l'aspartyle protéase traduisent clairement l'activité protéolytique du Penicillium (Le Bars et Gripon, 1981 ; Trieu-Cuot et al., 1982 a et b). Ces deux bandes cnt une intensité équivalente en début d'affinage mais par la suite $(\beta \mathrm{mpla}+\beta \mathrm{mp} 1 \mathrm{~b})$ diminue alors que $(\beta \mathrm{mp} 2 \mathrm{a}+\beta \mathrm{ap} 1)$ reste inchangée ou s'intensifie légèrement indiquant que les peptides correspondant sont produits en quantité appréciable et qu'ils ne sont pas ou peu dégradés. Après 59 jours d'affinage, la plupart des bandes ont diminué d'intensité, traduisant ainsi la forte protéolyse subie par le caillé. Seule la bande $(\beta \mathrm{mp} 2 \mathrm{a}+\beta \mathrm{ap} 1)$ n'a pas ou peu diminué d'intensité. Il est à noter que, mis à part le temps 59 jours, les diagrammes observés sont très voisins de ceux obtenus au cours de l'affinage de camemberts (Trieu-Cuot, 1981 ; Trieu-Cuot et Gripon, 1982). 


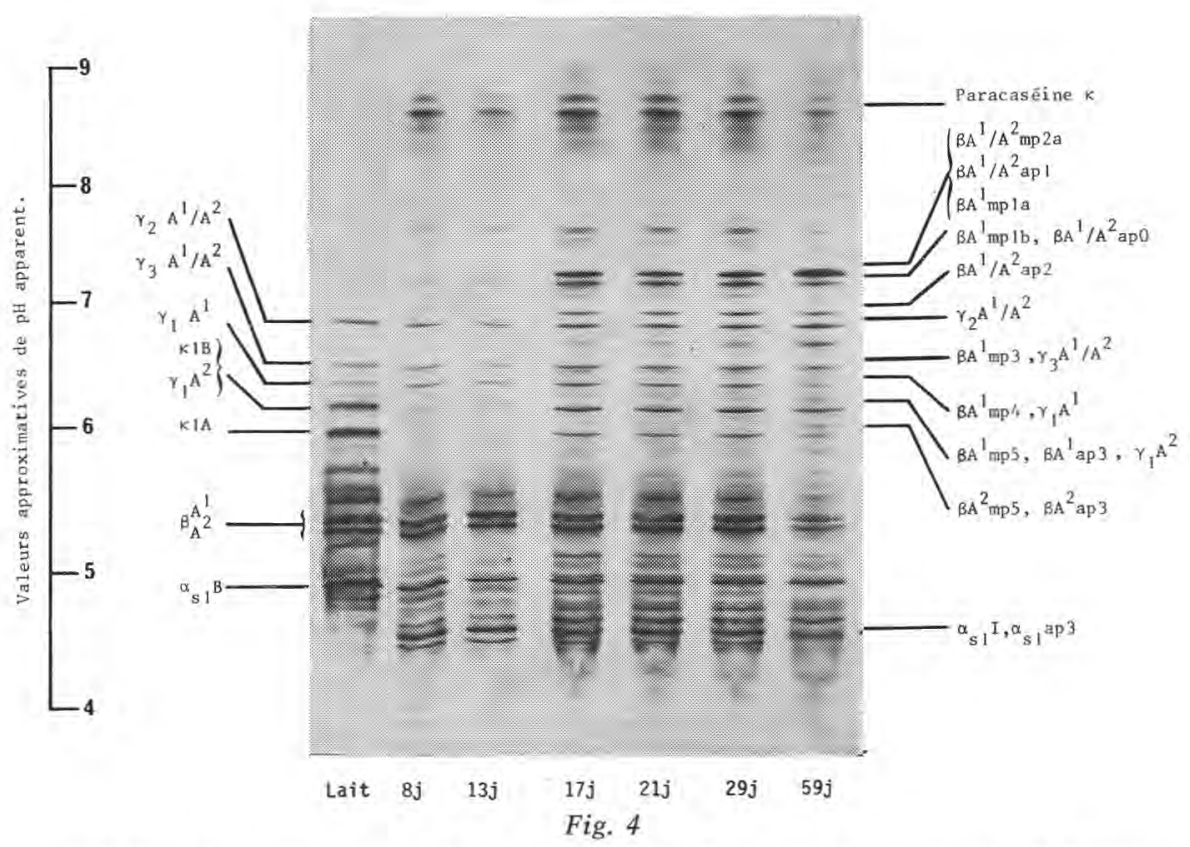

Evolution des diagrammes de focalisation isoélectrique au cours de l'affinage (fraction insoluble à $\mathrm{pH} 4,6$ ). Afin d'alléger la légende, certains des produits de dégradation issus du variant $\mathrm{A}^{2}$ de la caséine $\beta$ n'ont pas été indiqués sur la figure.

Development of isoelectric focusing patterns during bleu d'Auvergne ripening ( $\mathrm{pH} 4,6$ insoluble proteins). Some minor degradation products from $\beta A^{2}$-casein are not mentioned.

\section{Etude par focalisation isoélectrique}

Le pouvoir de résolution de la focalisation isoélectrique permet de mettre en évidence des bandes non détectées par électrophorèse à $\mathrm{pH}$ alcalin. Il est possible de distinguer le variant $\beta \mathrm{A}^{1} \mathrm{du}$ variant $\beta A^{2}$ et de ce fait le diagramme obtenu par focalisation isoélectrique (fig. 4) est beaucoup plus complexe que celui obtenu par électrophorèse. Sur la figure 3 nous avons identifié les différentes caséines présentes dans le lait de fabrication ainsi que les produits de dégradation majeurs dus à l'action de la métalloprotéase et de l'aspartyle protéase sur les caséines $\beta \mathrm{A}^{1}$ et $\beta \mathrm{A}^{2}$. Ces identifications résultent de travaux antérieurs (Trieu-Cuot et al., 1982 a et b). Toutefois, en dépit de cette bonne résolution, les bandes majeures résultant de l'action des 2 protéases de Penicillium se superposent, soit entre elles, soit avec les caséines $\gamma$ (fig. 3). En effet, le produit de dégradation majeur issu de l'action de l'aspartyle protéase sur la caséine $\beta\left(\beta\left(\mathrm{A}^{1} / \mathrm{A}^{2}\right)\right.$ ap1) a le même point isoélectrique que deux 
peptides issus de l'action de la métalloprotéase sur cette même caséine $\beta A^{1} \mathrm{mp} 1 \mathrm{a}$ et $\left.\beta\left(\mathrm{A}^{1} / \mathrm{A}^{2}\right) \mathrm{mp} 2 \mathrm{a}\right)$. Les autres produits $\beta \mathrm{A}^{1} \mathrm{mp} 3$, $\beta A^{1} m p 4$ et $\beta A^{1} m p 5$ comigrent respectivement avec $\gamma_{3} A^{1} / A^{2}, \gamma_{1} A^{1}$, $\gamma_{1} A^{2}$ et $\beta A^{1}$ ap3 (fig. 3) (Trieu-Cuot et Gripon, 1981 b ; Trieu-Cuot, 1981). Notons, cependant, que $\gamma_{2}$ résultant de l'action de la plasmine sur la caséine $\beta$, ne se chevauche avec aucun autre peptide. Cette bande caractérise donc l'action de cette enzyme dans le caillé. Elle ne s'intensifie pas ou peu au cours de l'affinage, contrairement à ce qui avait été observé pour des fromages de camembert.

\section{Etude par électrophorèse bidimensionnelle}

Après 17 jours d'affinage, les deux bandes alcalines majeures observées en focalisation $\left(\beta\left(\mathrm{A}^{1} / \mathrm{A}^{2}\right) \mathrm{mp} 2 \mathrm{a}+\beta\left(\mathrm{A}^{1} / \mathrm{A}^{2}\right)\right.$ ap $\left.1+\beta \mathrm{A}^{1} \mathrm{mp} 1 \mathrm{a}\right)$ et $\left(\beta A^{1} m p 1 b+\beta A^{1} / A^{2} a p 0\right)$, sont, en fait, essentiellement constituées des spots $\beta A^{1} \mathrm{mpla}, \beta \mathrm{A}^{1} \mathrm{mp} 1 \mathrm{~b}$ (spots 9 et 10 ) et $\beta\left(\mathrm{A}^{1} / \mathrm{A}^{2}\right)$ ap1 (spot 11 ). Ultérieurement l'intensité de coloration des deux spots résultant de la métalloprotéase ( $\beta A^{1} \mathrm{mpla}$ et $\left.\beta A^{1} \mathrm{mp} 1 \mathrm{~b}\right)$ diminue nettement alors que celle du spot résultant de l'aspartyle protéase $\left(\beta\left(\mathrm{A}^{1} / \mathrm{A}^{2}\right)\right.$ ap1) s'intensifie indiquant que cette enzyme poursuit son action. Après 59 jours, tous les spots mis à part $\beta\left(A^{1} / A^{2}\right)$ ap1 ont diminué d'intensité, les caséines $\alpha_{s 1}$ et $\beta$ ont pratiquement disparu.

Au cours de l'affinage, les différents spots 7 et 8 correspondant aux caséines $\gamma_{2}$ et $\gamma_{3}$ ne s'intensifient pas, confirmant ainsi les résultats de la focalisation isoélectrique.

In vitro, la chymosine (constituant majeur de la présure) hydrolvse successivement la caséine $\beta$ en 3 zones distinctes et conduisant à l'apparition de 3 produits de dégradation $\beta I, \beta I I, \beta I I I$ (Creamer, 1973). Aucun de ces produits de dégradation n'est observé au cours de l'affinage du bleu d'Auvergne.

\section{DISCUSSION}

L'action de la chymosine se détecte nettement dès le début l'affinage par l'apparition du composé $\alpha_{S_{1}}$ I caractéristique de cette enzyme et correspondant à la coupure des liaisons $\mathrm{Phe}_{23}$ - $\mathrm{Phe}_{24}$ et Phe $_{24}-\mathrm{Val}_{25}$ (Hill et al., 1974 ; Creamer et Richardson, 1974 ; Pelissier et al., 1974).

Après des études électrophorétiques, Godinho et Fox (1982) ont suggéré que, dans les pâtes persillées, l'hydrolyse de la caséine $\alpha_{\text {s1 }}$ était principalement due à la présure. Bien que le $\mathrm{pH}$ d'une pâte persillée (tab. 1) soit plus favorable à l'action de la présure qu'il ne l'est dans un camembert (Noomen, 1978 a), il nous paraît difficile de soutenir cette affirmation au vu de simples études électrophorétiques. 
En effet, l'hydrolyse de la caséine $\alpha_{s_{1}}$ par la métalloprotéase ou l'aspartyle protéase de $P$. roqueforti conduit à l'apparition de peptides ayant une mobilité voisine de celle des peptides $\alpha_{\mathrm{s}_{1} \mathrm{I}}$, $\alpha_{s_{1}} \mathrm{~V}, \alpha_{s_{1}} \mathrm{VII} / \mathrm{VIII}$ obtenus après action de la chymosine (Mulvihill et Fox, 1980). Plus particulièrement, il est probable qu'après le développement du Penicillium, l'aspartyle protéase qui hydrolyse la caséine $\alpha_{s_{1}}$ en un peptide $\alpha_{\mathbb{s}_{1}}$ ap3 de même point isoélectrique et poids moléculaire que $\alpha_{s_{1}} \mathrm{I}$ (Trieu-Cuot et al., 1982 b) participe à l'intensification de la bande correspondante.

Comme il a été constaté pour d'autres fromages (Ledford et al., 1966), nous n'avons pu mettre en évidence par aucune des 3 techniques utilisées, la présence des produits $\beta I, \beta I I, \beta I I I$, obtenus après hydrolyse " in vitro » de la caséine $\beta$ par la présure.

Creamer (1975) a suggéré que, dans les fromages, les liaisons correspondant aux peptides $\beta I, \beta I I, \beta I I I$ ne sont pas accessibles à l'enzyme. Elles sont, en effet, situées dans la partie C-terminale de la molécule qui participerait à la structure du réseau protéique du caillé par la formation de liaisons hydrophobes.

L'électrophorèse à $\mathrm{pH}$ alcalin et l'électrophorèse bidimensionnelle révèlent bien la très forte protéolyse obtenue après 59 jours d'affinage dans les pâtes persillées. La quasi totalité des produits de dégradation des caséines sont hydrolysés plus avant et voient leur intensité de coloration diminuer. Seul Bap1, le produit de l'hydrolyse de la caséine $\beta$ par l'aspartyle protéase (spot $n^{\circ} 11$ ) est particulièrement résistant (cf. fig. 5).

Par les trois techniques électrophorétiques utilisées, on constate clairement que les diagrammes obtenus sont extrêmement voisins de ceux observés avec le camembert (Trieu-Cuot et Gripon, 1982). En électrophorèse bidimensionnelle, tous les spots de forte ou moyenne intensité détectés avec un échantillon de bleu d'Auvergne, ont un spot homologue dans les diagrammes obtenus avec le camembert. Ceci est constaté aussi bien dans la zone alcaline du gel (contenant principalement des produits d'hydrolyse de la caséine $\beta$ ) que dans la partie acide (contenant principalement des produits d'hydrolyse de la caséine $\alpha_{s_{1}}$ ). On observe donc clairement que, qualitativement, la protéolyse dans les deux types de fromages est extrêmement voisine et reflète bien l'homologie déjà observée au niveau des systèmes protéolytiques de ces deux moisissures (Trieu-Cuot et al., 1982 a et b).

Il est cependant possible de mettre en évidence deux différences.

Dans le cas des fromages à croûte fleurie (Trieu-Cuot et Gripon, 1982), on observe en fin d'affinage, une intensification des bandes (ou des spots en électrophorèse bidimensionnelle) correspondant aux produits d'hydrolyse de la caséine $\beta$ par la plasmine (spots 4,5 , 7 et 8) révélant ainsi une activité plus importante de cette dernière. Le même phénomène n'est pas observé avec le bleu d'Auvergne et 


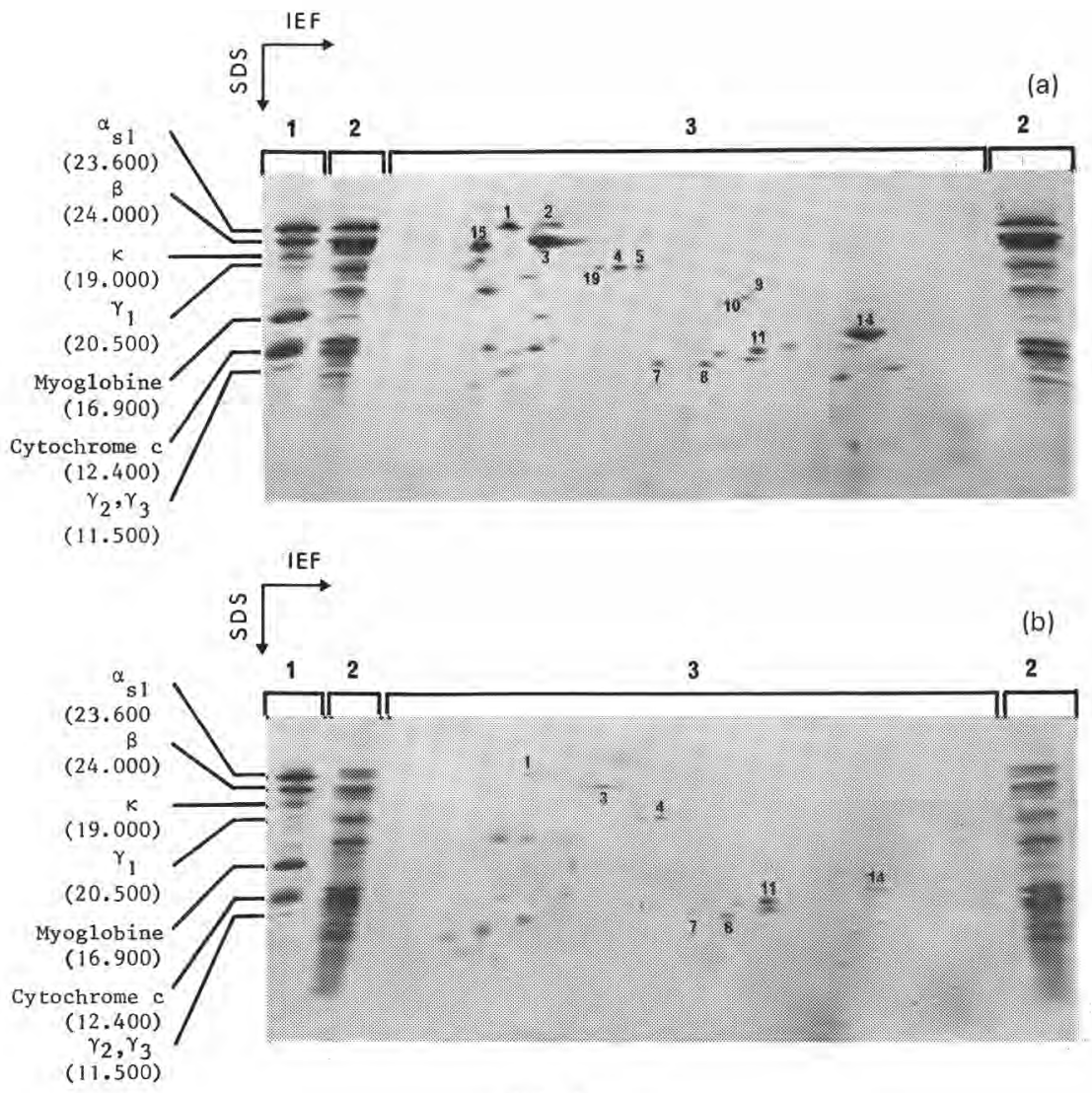

Fig. 5

Electrophorèse bidimensionnelle (3) de la fraction insoluble à pH 4,6 de fromages de bleu d'Auvergne après 29 (a) et 59 (b) j d'affinage.

Identification des spots : 1 , caséine $\alpha_{S_{1}} ; 2$, caséine $\alpha_{s_{2}} ; 3$, caséines $\beta A^{1}$ et $\beta A^{2}$; $4, \gamma_{1} \mathrm{~A}^{2} ; 5, \gamma_{1} \mathrm{~A}^{1} ; 7, \gamma_{3} \mathrm{~A}^{1} / \mathrm{A}^{2} ; 8, \gamma_{2} \mathrm{~A}^{1} / \mathrm{A}^{2} ; 9, \beta \mathrm{A}^{1} \mathrm{mpla} ; 10, \beta \mathrm{A}^{1} \mathrm{mplb}$; 11, $\beta \mathrm{A}^{1} / \mathrm{A}^{2} \mathrm{ap} 1 ; 14$, para-caséine $\mathrm{K} ; 15, \alpha_{\mathrm{s}_{1}} \mathrm{I}+\alpha_{\mathrm{s}_{1}} \mathrm{ap} 3$.

(1) et (2) représentent respectivement les électrophorèses SDS des protéines étalons et de l'échantillon étudié.

2-dimensional electrophoresis (3) of the $\mathrm{pH} 4.6$ insoluble fraction of bleu d'Auvergne after 29 (a) and 59 (b) days of ripening.

Identification of the spots: $1: \alpha_{S_{1}}$-casein; $2: \alpha_{s_{2}}$-casein; $3: \beta A^{I}$ and $\beta A^{2}$-caseins; $4: \gamma_{1} A^{2}$; 5: $\gamma_{1} A^{1} ; 7: \gamma_{3} A^{t} / A^{2} ; 8: \gamma_{2} A^{1} / A^{2} ; 9: \beta A^{1}$ mpla; 10: $\beta A^{2}$ mplb; 11: $\beta A^{1} / A^{2} a p l ; 14:$ paracasein-K; 15: $\alpha_{s_{1}} I+\alpha_{s_{1}}$ ap3.

(1) and (2) respectively represent SDS electrophoresis of molecular weight marquers and cheese sample. 
l'explication peut être recherchée dans l'évolution différente du $\mathrm{pH}$ de ces fromages. Alors qu'en fin d'affinage le $\mathrm{pH}$ du bleu d'Auvergne n'excède pas 6,0-6,2, il atteint une valeur de 7,0 en fin de maturation en surface d'un camembert, favorisant ainsi une activité accrue de la plasmine (Noomen, 1978 b).

Dans le bleu d'Auvergne, les produits résultant de l'activité de l'aspartyle protéase et de la métalloprotéase apparaissent ensemble immédiatement après la croissance du Penicillium roqueforti. Il semble donc que les deux protéases soient synthétisées et libérées simultanément dans le caillé au cours du développement du Penicillium. Dans le cas des fromages à croûte fleurie, on a nettement observé un décalage dans la production de ces deux protéases dans le fromage. L'activité de la métalloprotéase est détectable dès 7 jours après la fabrication alors que les produits de l'aspartyle protéase ne sont observés que 10 jours après la fabrication.

L'évolution de la texture des fromages est généralement attribuée à la protéolyse et on constate, en effet, que l'addition de protéases dans les caillés entraîne des modifications des paramètres rhéologiques (Vassal et al., 1982; Law et Wigmore, 1982). La consistance des pâtes persillées est différente de celle des fromages à croûte fleurie, elle ne présente pas l'assouplissement, voir l'écoulement observé dans un camembert affiné. Il apparaît difficile d'imputer ces différences de texture à une différence de protéolyse. Qualitativement, cette dernière est sensiblement la même dans les deux types de caillés. De plus, la protéolyse est plus poussée dans les pâtes persillées affinées et l'on pourrait s'attendre à ce que la pâte soit plus souple et plus coulante dans ces dernières, ce qui n'est pas le cas. Il est donc très probable que d'autres facteurs que la protéolyse influent sur la texture des deux types de fromages et conditionnent son évolution. On peut noter que les paramètres physico-chimiques de ces caillés sont différents, le camembert ayant une pâte plus humide, plus déminéralisée et de $\mathrm{pH}$ plus élevé (en fin d'affinage) que les pâtes persillées.

\section{Remerciements}

Nous remercions vivement la fromagerie Centre-Lait (Aurillac) pour la fabrication des fromages ainsi que M. Pradel pour le prélèvement des échantillons.

\section{Bibliographie}

Creamer (L. K), Mills (O. E.) and Richards (E. L.) (1971). - The action of rennets on the caseins. I. Rennin action on $\beta$-casein-B in solution. J. Dairy Res., 38, 269-280.

CREAMER (L. K.) and RichaRdson (B. C.) (1974). - Identification of the primary degradation product of $\alpha_{s_{1}}$-casein in Cheddar cheese. $N_{d} Z$. J. Dairy Sci.: Technol., 9, 9-13. 
Desmazeaud (M. J.), Gripon (J. C.), Le Bars (D.) et Bergère (J. L.) (1976). Etude du rôle des micro-organismes et des enzymes au cours de la maturation des fromages. III. Influence des micro-organismes (Streptococcus lactis, Penicillium caseicolum, Penicillium roqueforti). Le Lait, 56, 379-396.

Devoyod (J. J.), Bret (G.) et Auclatr (J.E.) (1968). - La flore microbienne du fromage de Roquefort. I. Son évolution au cours de la fabrication et de l'affinage du fromage. Le Lait,, 48, 613-629.

Godinho (M.) and Fox (P. F.) (1982). - Ripening of blue cheese. Influence of salting rate on proteolysis. Milchwissenschaft, 37, 72-75.

Gripon (J. C.), Desmazeaud (M. J.), Le Bars (D.) et Bergère (J. L.) (1975). - Etude du rôle des micro-organismes et des enzymes au cours de la maturation des fromages. II. Influence de la présure commerciale. Le Lait, 55, 502-516.

HILL (R. P.), LAHAV (E.) and GIVol (D.) (1974). - A rennin-sensitive bond in $\alpha_{s_{1}}$ B-casein. J. Dairy Res., 41, 147-153.

LaW (B. A.) and Wigmore (A.) (1982). - Accelerated cheese ripening with food grade proteinases. J. Dairy Res., 49, 137-146.

LE BARS (D..) and GRIPON (J. C.) (1981). - Role of Penicillium roqueforti proteinases during blue cheese ripening. J. Dairy Res., 48, 479-487.

LedFord (R.A.), O'Sullivan (A. C.) and Nath (K. R.) (1966). - Residual casein fractions in ripened cheese determined by polyacrylamide-gel electrophoresis. J. Dairy Sci., 49, 1098-1101.

LeNoIR (J.) (1962), - Sur la dégradation des protides au cours de la maturation du Camembert. C.R. Acad. Agr., 48, 160-169.

Mulvihill (D. M.) and Fox (P. F.) (1980). - Proteolysis of a s. $_{1}$-casein by chymosin in dilute $\mathrm{NaCl}$ solutions and in Cheddar cheese. Ir. J. Fd Technol., 4, 19-23.

Noomen (A) (1978a). - Activity of proteolyte enzymes in simulated soft cheeses (Meshanger type). 2. Activity of calf rennet. Neth. Miik Dairy J., 32, 49-68.

Noomen (A.) (1978b). - Activity of proteolytic enzymes in simulated soft cheeses (Meshanger type) 1. Activity of milk protease. Neth Milk Dairy J., 32, 26-48.

Pelissier (J. P.), Mercier (J. C.) et Ribadeau-Dumas (B.) (1974). - Etude de la protéolyse des caséines $\alpha_{S_{1}}$ et $\beta$ bovines par la présure. Spécificité d'action. Peptides amers libérés. Ann. Biol. Anim. Biochim. Biophys., 14, 343-362.

Trieu-Cuot (P.) (1981), - Etude des caséines et de leurs produits de dégradation par méthodes électrophorétiques. Application à l'étude de l'affinage du Camembert. Thèse Université Paris XI.

Trieu-Cuot (P.), Archieri-Haze (M. J.) et Gripon (J. C.) (1982a). - Etude comparative de l'action des métalloprotéases de Penicillium caseicolum et Penicillium roqueforti sur les caséines $\alpha_{\mathrm{s}_{1}}$ et $\beta$. Le Lait, 62, 234-249.

Trieu-Cuot (P.), Archieri-Haze (M. J.) and Gripon (J. C.) (1982b). - Effect of aspartyl proteinases of Penicillium caseicolum and Penicillium roqueforti on caseins. J. Dairy Res., 49, 487-500.

Trieu-CUOT (P.) and GRIPON (J. C.) (1981a). - Electrofocusing and two-dimensional electrophoresis of bovine caseins. J. Dairy Res., 48, 303-310.

Trieu-Cuot (P.) and Gripon (J. C.) (1981b). - Casein hydrolysis by Penicillium caseicolum and $P$. roqueforti proteinases: a study with isoelectric focusing and two-dimensional electrophoresis. Neth. Milk Dairy J., 35, 353-357.

Trieu-Cuot (P.) and GRIPon (J. C.) (1982). - A study of proteolysis during Camembert cheese ripening using isoelectric focusing and two-dimensional electrophoresis. J. Dairy Res., 49, 501-510.

Vassal (L.), Desmazeaud (M. J.) et Gripon (J. C.) (1982). - Modification de fromages à pâte pressée par addition d'une métalloprotéase de Micrococcus caseolyticus. 315-318 dans Utilisation des Enzymes en Technologie Alimentaire, édité par P. Dupuy, Lavoisier, Paris. 